KERNFORSCHUNGSZENTRUM

\author{
KARLSRUHE
}

Institut fur Angewandte Kernphysik

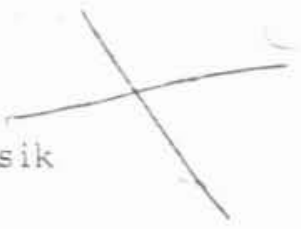

Schnelle Koinzidenzstufe mit Lawinentransistoren

W. Michaelis, H. Schmidt, C. Weitkamp

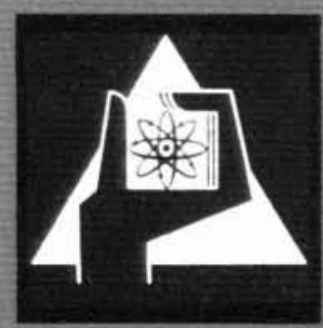

GESELLSCHAFT FUR KERNFORSCHUNG M.B.H. KARLSRUHE 


\title{
SCHNELLE KOINZIDENZSTUFE MIT LAWINENTRANSISTOREN
}

\author{
W. MICHAELIS, H. SCHMIDT und C. WEITKAMP
}

Institut für Angewandte Kernphysik, Kernforschungszentrum Karlsruhe

Eingegangen am 24. Mai 1964

A fast coincidence circuit using avalanche transistors is described. Excellent results can be obtained even with slow scintillators. In a fast slow coincidence assembly with $\frac{3}{2} " \varnothing \times 2^{\prime \prime} \mathrm{NaI}(\mathrm{Tl})$ crystals and RCA $6810 \mathrm{~A}$ photomultipliers a resolving time

\section{Einleitung}

Bei vielen Arbeiten auf dem Gebiet der Kernspektroskopie sind Koinzidenzschaltungen mit Auflösungszeiten im Nanosekundenbereich zu einem unentbehrlichen Hilfsmittel geworden. Neben den bewährten Röhrenschaltungen werden in neuerer Zeit auch eine Reihe schneller Koinzidenzstufen mit Halbleiter-Bauelementen verwendet. In den meisten dieser Schaltungen erfolgen Normierung und Impulsformung entweder durch Zusteuerung eines leitenden Transistors in Verbindung mit einem Clipping-Kabel oder durch eine Tunneldiode, die durch ein RCoder LC-Glied oder durch eine besondere Schaltung zurückgesetzt wird. Die für organische Szintillatoren entwickelten Anordnungen dieser Art haben sich in der Regel gut bewährt, während für $\mathrm{NaJ}(\mathrm{Tl})$ der relativ enge Aussteuerbereich dieser Schaltungen und die lange Abklingzeitkonstante des Szintillators Schwierigkeiten bereiten. of $1.6 \mathrm{nsec}$ at nearly $100 \%$ coincidence efficiency was realized for the $\mathrm{Co}^{60}$ gamma ray cascade. The circuit is stable and reliable.

Da Lawinentransistoren sehr kurze und stromstarke Impulse mit gut definiertem, weitgehend amplitudenunabhängigem Einsatzpunkt erzeugen können, liegt es nahe, diese Transistoren als Bauelemente für eine schnelle Koinzidenzstufe zu verwenden. Es wurde eine Schaltung entwickelt. die für $\mathrm{NaJ}(\mathrm{Tl})$ als Szintillator geeignet ist, die ein gutes Koinzidenzauflösungsvermögen und einen großen Aussteuerbereich hat und die sich auch für Mehrfach-Koinzidenzen verwenden läßt.

\section{Schaltung und Arbeitsweise}

Die Schaltung geht aus Abb. 1 hervor. Sie läßt sich aufgliedern in Normierstufen, Impulsformerstufen, die eigentliche Koinzidenzstufe und einen Ausgangskreis.

\subsection{Normierstufe}

Jede Normierstufe besteht aus einem Lawinentransistor T1 in Emitter-Schaltung, den die negativen

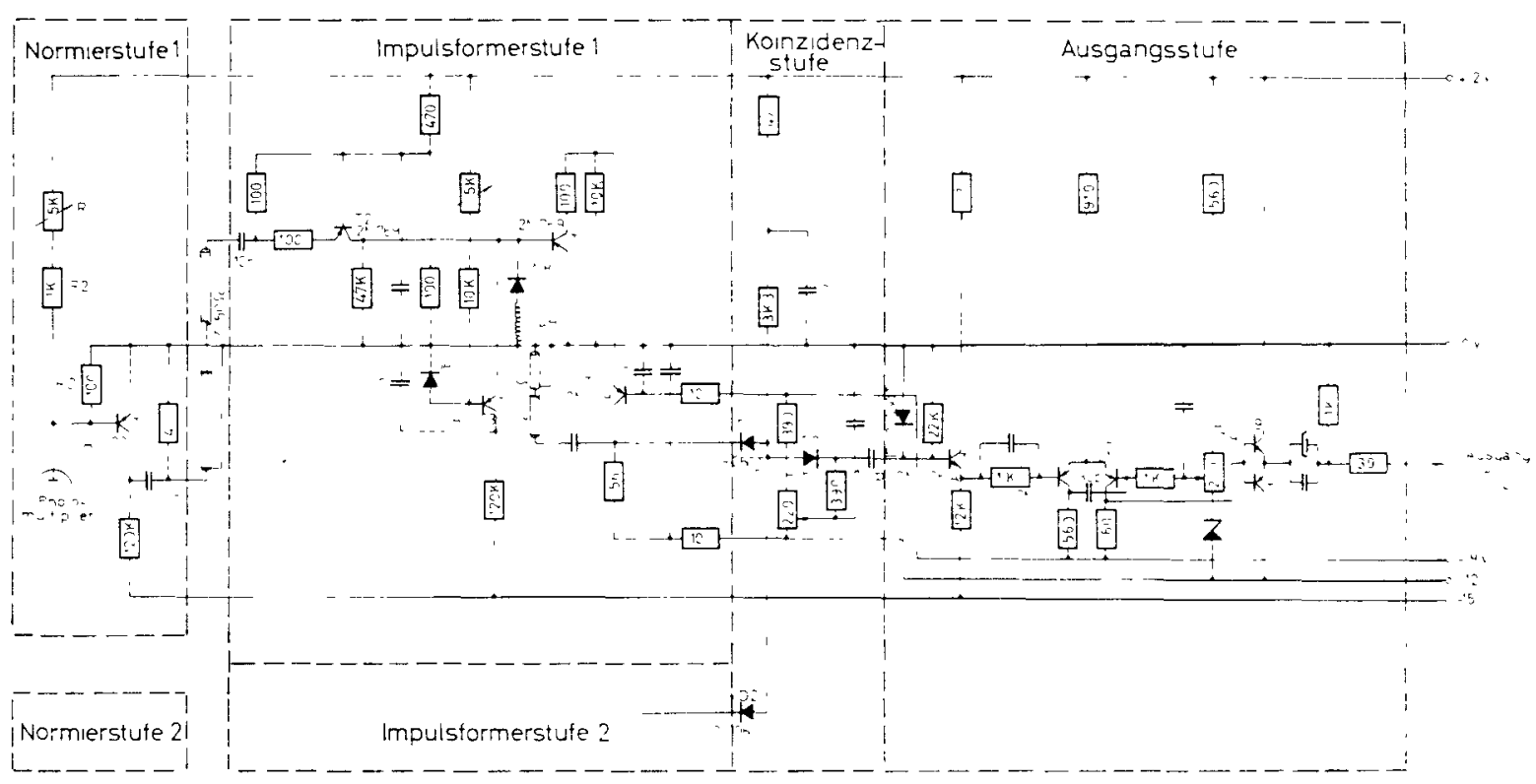

Abb. 1. Schaltung. C : induktionsarme Blockkondensatoren. 
Stromimpulse des Detektors in das Durchbruchsgebiet steuern (über die Wirkungsweise von Lawinentransistoren vgl. z.B. Ref. ${ }^{1}$ )). Dadurch wird das Abfließen der im Kondensator $\mathrm{Cl}$ gespeicherten negativen Ladung ermöglicht. Es entsteht am Ausgang ein positiver Nadelimpuls mit einer Anstiegszeit von etwa $1 \mathrm{~ns}$, einer Breite von einigen ns und einer Amplitude von ungefähr $1 \mathrm{~V}$ (Abb. 2a). Man erhält
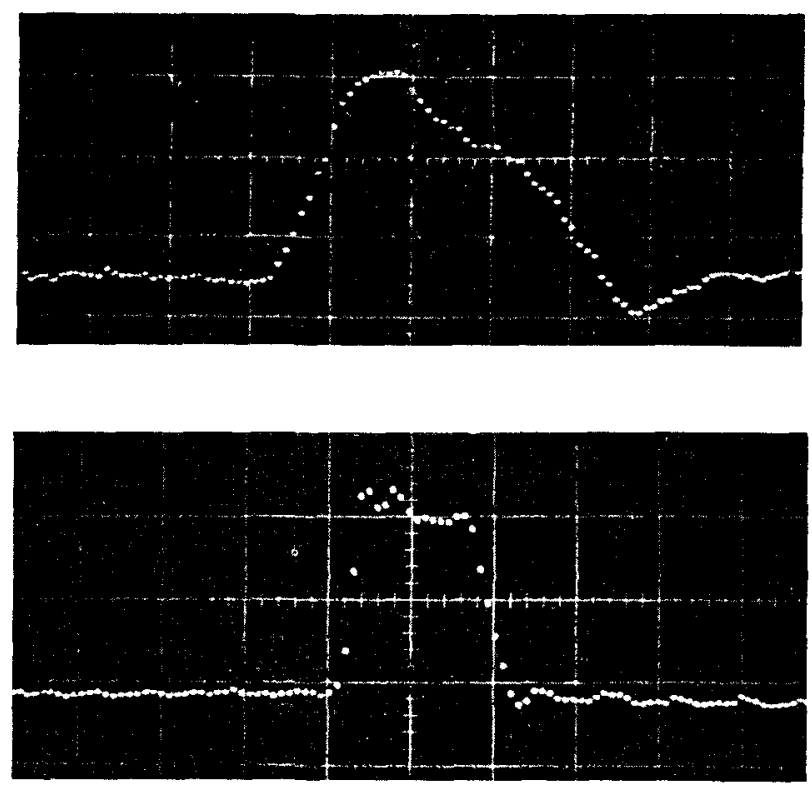

Abb. 2. (a) Ausgangssignal der Normierstufe. Amplitudenskala $0.5 \mathrm{~V} / \mathrm{cm}$; Zeitablenkung $2 \mathrm{~ns} / \mathrm{cm}$. (b) Ausgangssignal der Impulsformerstufe. Amplitudenskala $1 \mathrm{~V} / \mathrm{cm}$; Zeitablenkung $5 \mathrm{~ns} / \mathrm{cm}$. Anstiegszeit des Oszillographen $0.6 \mathrm{~ns}$.

so ein stromstarkes Zeitsıgnal, das über ein $50 \Omega-\mathrm{Kabel}$ zum Eingang der Impulsformerstufe geführt wird. Im Gegensatz zu den üblichen Begrenzer-Schaltungen liefert der Transistor T1 keine Signale, deren Amplitude unter der Standard-Impulshöhe liegt. Wie bei Tunneldioden-Schaltungen ist die Ansprechschwelle der Normierstufe variabel, ein Vorteil, der zur Diskriminierung der Detektorsignale vor der Koinzidenzstufe ausgenutzt werden kann. Die Ansprechschwelle läßt sich mit den Widerständen R2 und R3 grob einstellen. Die Feinregulierung erfolgt mit dem Potentiometer R1. In der angegebenen Schaltung sind die Widerstände so dimensioniert, daß die Schwelle zwischen $100 \mathrm{mV}$ und $1.2 \mathrm{~V}$ (an ungefähr $66 \Omega$ ) variiert werden kann. Der Aussteuerbereich ist beim Lawinentransistor wesentlich größer als bei Tunneldioden. Lediglich die relativ lange Totzeit der Einheit, die in der Größenordnung $1 \mu$ s liegt, kann bei hohen Zählraten stören. Dieser Nachteil läßt sich jedoch mit wenig Aufwand durch eine von Stuckenberg1) vorgeschlagene Abänderung der Schaltung beheben.

\subsection{IMPULSFORMERSTUFE}

Die Impulsformerstufe ist eine Weiterentwicklung einer erstmals von Bramson ${ }^{2}$ ) vorgeschlagenen Anordnung zur Erzeugung schneller Rechtecksignale. Die positiven Impulse der Normierstufe werden durch den in Basisschaltung arbeitenden Transistor T2 eingekoppelt. Sie steuern den Lawinentransistor T3 über dessen Emitter ins Durchbruchsgebiet und erlauben so das Abfließen der im Kondensator C2 gespeicherten Ladung. Da der Basiskreis von T3 hochohmig ist, fließt der größte Teil der Ladung von C2 in den Emitter und erzeugt so einen etwa 4 ns breiten, stromstarken negativen Impuls, der den nachfolgenden Transistor T4 in die Sättigung steuert. Eine Drossel im Basiskreis dieses Transistors verhindert ein direktes Abfließen der Ladung. Das am Kollektor von T4 entstehende positive Signal sättigt einen weiteren Transistor T5 hinreichend stark, so daß dessen Kollektorimpuls durch ein kurzgeschlossenes ClippingKabel zu einem Rechtecksignal mit Längen bis zu ungefähr $50 \mathrm{~ns}$ geformt werden kann. Die Breite dieser Signale bestimmt das zeitliche Auflösungsvermögen der Koinzidenzstufe. Für große Impulslängen sollen die Transistoren T4 und T5 eine Stromverstärkung $\beta \approx 200$ besitzen.

T5 liefert einen Impulsstrom von etwa $100 \mathrm{~mA}$. Die Anstiegszeit beträgt weniger ais $1 \mathrm{ns,} \mathrm{die} \mathrm{Rück-}$ flanke hängt leicht von der Impulslänge ab und liegt zwischen 1 und 2 ns (Abb. 2b). Der Kollektorwiderstand ist zugleich Abschlußwiderstand des ClippingKabels und Bestandteil des Spannungsteilers im Koinzidenzkreis.

\subsection{KOINZIDENZSTUFE}

Die Koinzidenzstufe ist eine Rossi-Diodenschaltung, an deren Ausgang das logische Produkt der Eingänge erscheint. Diese Schaltung wurde einer schnellen Addierstufe vorgezogen, da sie sich wesentlich besser zu einem Mehrfachkoinzidenzkreis erweitern läßt und geringere Anforderungen an die Stabilität der übrigen Bauelemente stellt. Sie kann zudem durch Verwendung von npn-Transistoren oder durch Betrieb des Transistors T5 in Emitterschaltung in einfacher Weise zu einem Koinzidenz-Antikoinzidenzkreis erweitert werden. Die verwendeten Dioden (D1, D2) vom Typ ID 10 - $050 \mathrm{~T}$ haben eine Schalizeit von $\leqslant 0.5 \mathrm{~ns}$. Das günstige Amplitudenverhältnis für 
Koinzidenz- und Einzelsignale (etwa $12: 1$ ) wird durch eine vorgespannte Diode (D3) als nichtlineares Schaltelement weiter verbessert.

\subsection{Ausgangsstufe}

Die Koinzidenzsignale gelangen über den als Emitterfolger arbeitenden Transistor T6 auf einen Univibrator (T7, T8), der aus Transistoren mit kurzer Schaltzeit aufgebaut ist und auf Signale mit Impulsbreiten von nur wenigen ns anspricht. Eine im Ruhezustand leitende Diode (D4) im Basiskreis von T6 stabilisiert den Spannungspegel am Eingang des Univibrators. Die Einstellung der Triggerschwelle erfolgt mit dem Potentiometer R6. Temperatureinflüsse über den Kollektorsperrstrom werden durch eine symmetrische Dimensionierung der Basiskreise des Univibrators klein gehalten. Für die Eingangssignale ist der Basiswiderstand von T7 kapazitiv überbrückt. Der Innenwiderstand der Basis-EmitterDiodenstrecke von T6 kompensiert in etwa den Widerstand im Spannungsteiler der Basis von T8.

Die positiven Rechtecksignale des Univibrators gelangen über einen Emitterfolger (T9, T10) zum Ausgang. Sie haben bei $50 \Omega$ Lastwiderstand eine Amplitude von $4 \mathrm{~V}$, ihre Breite beträgt $0.5 \mu \mathrm{s}$.

\section{Testergebnisse}

Messbare Temperatureffekte sind lediglich bei der Ansprechschwelle der Normierstufe zu beobachten. Bei Zimmertemperatur beträgt die Instabilität etwa $1 \%$ des Schwellwertes pro ${ }^{\circ} \mathrm{C}$ Temperaturänderung.

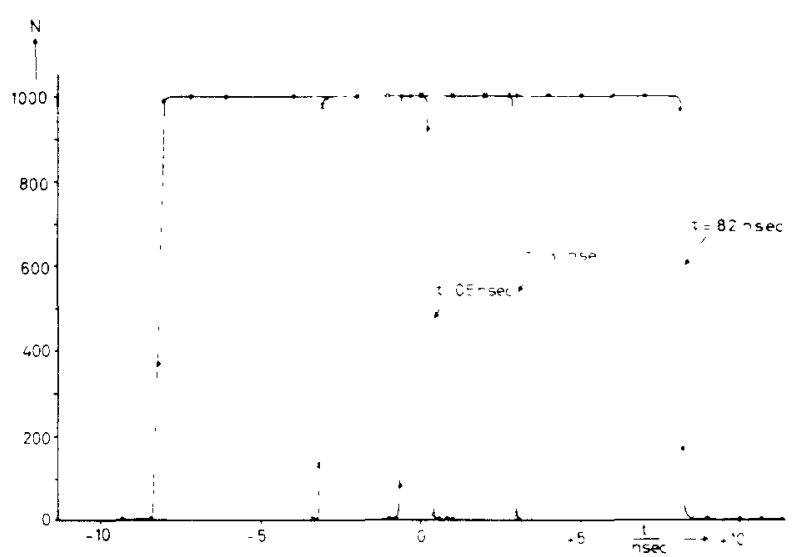

Abb. 3. Koinzidenzzahlrate $N$ als Funktion der relativen Verzögerung $t$ fur künstliche Impulse. Abstand der Messpunkte auf den Flanken 0.15 ns.
Wird die Normierstufe mit Impulsen angesteuert, deren Amplitude sich um einen Faktor 100 ändert, so schwankt die Höhe der Ausgangssignale um $\pm 12 \%$ des Mittelwertes. Amplitudenschwankungen von dieser Größe haben keinerlei Einfluß auf die Funktion der nachfolgenden Stufen. Auf Grund ihrer Arbeitsweise ist die beschriebene Koinzidenzschaltung unempfindlich gegenüber Störungen durch parasitäre Impulse.

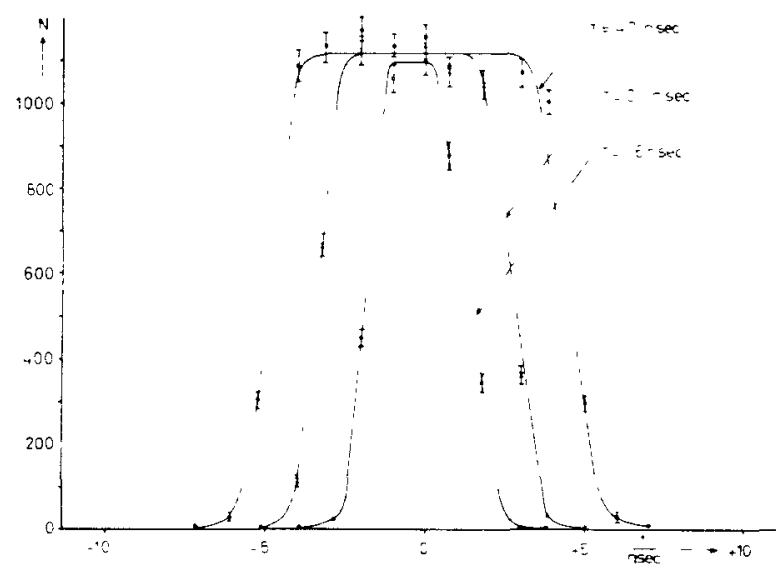

Abb. 4. Koinzidenzzahlrate $N$ als Funktion der relativen Verzogerung $t$ fur Szintillationsimpulse der $\mathrm{Co}^{60}-\hat{\gamma}-\hat{-},-$ Kaskade, aufgenommen mit $\frac{3}{2} " \emptyset \times 2 " \mathrm{NaJ}(\mathrm{T} 1)-\mathrm{K}$ ristallen und Photomultipliern vom Typ RCA $6810 \mathrm{~A}$.

Das Koinzidenzauflösungsvermögen wurde sowohl mit einem Impulsgenerator als auch mit einer radioaktiven Quelle untersucht. Abb. 3 zeigt die Meßergebnisse für künstliche Impulse bei Auflösungszeiten von $0.5,3.1$ und 8.2 ns. Für die Messungen mit einer natürlichen Quelle wurde ein $\mathrm{Co}^{60}-$ Präparat benutzt. Die Detektoren bestanden aus $\frac{3}{2} " \varnothing \times 2 " \mathrm{NaJ}$ (T)-Kristallen und Photomultipliern vom Typ RCA 6810 A. Die Auflösungskurven einer SchnellLangsam-Koinzidenz mit Auflösungszeiten von 1.6, 3.1 und $4.7 \mathrm{~ns}$ sind in Abb. 4 wiedergegeben. Bei $1.6 \mathrm{~ns}$ beträgt der Koinzidenzwirkungsgrad noch nahezu $100 \%$. Dieses Ergebnis entspricht in etwa dem theoretisch optimal möglichen Auflösungsvermögen für die $\mathrm{Co}^{60}$-Kaskade bei Verwendung von $\mathrm{NaJ}(\mathrm{Tl})$ als Szintillator. Es ist zu erwarten, daß die beschriebene Schaltung für Detektoren mit einem günstigeren Zeitverhalten noch bessere Resultate liefert.

\section{References}

1) M.I. Stuckenberg, DESY-Notiz A 2.92 (1962).

2) M. Bramson, IRE Trans. Nucl. Science NS 9, No. 4 (1962) 35. 